03

\title{
Исследование гидродинамического сопротивления щелевого микроканала с текстурированной стенкой
}

\author{
(C) А.С. Лобасов ${ }^{1,2}$, А.В. Минаков ${ }^{1,2}$ \\ ${ }^{1}$ Сибирский федеральный университет, Красноярск, Россия \\ ${ }^{2}$ Институт теплофизики им. С.С. Кутателадзе СО РАН, Новосибирск, Россия \\ E-mail: perpetuityrs@mail.ru
}

Поступило в Редакцию 12 мая 2021 г.

В окончательной редакции 10 сентября 2021 г.

Принято к публикации 19 сентября 2021 г.

\begin{abstract}
Представлены результаты численных исследований коэффициента гидродинамического трения щелевого микроканала с текстурированной поверхностью, а также перепада давлений в таком канале и эффективной длины скольжения на стенке для различных чисел Рейнольдса. Высота канала равнялась $10 \mu \mathrm{m}$, а длина варьировалась от 25 до $500 \mu \mathrm{m}$. Установлено, что перепад давлений в текстурированном микроканале был меньше, чем в обычном канале для любой его длины. Получены зависимости относительного перепада давлений, коэффициента гидродинамического трения и эффективной длины скольжения от числа Рейнольдса для различных длин канала. Предложена корреляция, описывающая зависимость относительного перепада давлений от числа Рейнольдса для малых длин канала. Коэффициент гидродинамического трения описывается корреляцией вида 20/Re.
\end{abstract}

Ключевые слова: микроканал, текстурированная стенка, скольжение на стенке, численное моделирование.

DOI: 10.21883/PJTF.2022.01.51872.18866

В последнее время активно развивается наногидродинамика вследствие миниатюризации устройств в различных областях техники: аэрокосмической индустрии, энергетике, электронике, транспорте и медицине, нефтегазовой отрасли. Кроме того, мини- и микроканалы широко распространены в биологических системах. Однако в микроканалах, где поперечные размеры очень малы, т.е. стенки расположены очень близко друг к другу, при выполнении условия прилипания скорость движения жидкости также очень мала, а значения гидравлических сопротивлений велики. Одним из возможных решений данной проблемы является использование явления скольжения на стенке, которое заключается в том, что в силу определенных поверхностных свойств скорость жидкости у стенок становится отличной от нуля. Одним из интересных направлений исследований является изучение поверхностей с нанесенными микротекстурированными слоями с предельно высокой или предельно низкой смачиваемостью и дальнейшее управление смачиваемостью этих наноструктур. В последние годы появилась возможность создавать микротекстурированные материалы с контактными углами порядка $150^{\circ}$ с эффектом самоочистки, водоотталкивания, антиобледенения и противозапотевания [1]. Также в настоящее время продолжают разрабатываться различные микрофлюидные приборы: микромасштабные теплофизические устройства, био-МЭМС и ,лаборатории на чипе ${ }^{\text {“ }}[2,3]$, использующие ультрагидрофобные свойства. Поэтому в последние годы проявляется огромный интерес к исследованию высоко- и супергидрофобных микротекстурированных поверхностей как за рубежом [1-5], так и в России [6-10].
В связи с этим в настоящей работе исследовано влияние длины щелевого текстурированного микроканала на перепад давлений в таком канале, коэффициент гидродинамического сопротивления и эффективную длину скольжения на стенке для различных чисел Рейнольдса. Высота микроканала была равна $10 \mu \mathrm{m}$, длина варьировалась в пределах от 25 до $500 \mu \mathrm{m}$. В работе рассматриваются трехмерные течения несжимаемых жидкостей, для описания которых используется гидродинамический подход, основанный на решении системы уравнений Навье-Стокса. Подробно это подход рассмотрен в работе [11], далее же отмечены основные моменты численной методики. Разностный аналог конвективно-диффузионных уравнений находится с помощью метода конечного объема для структурированных многоблочных сеток. Аппроксимация конвективных членов уравнений переноса осуществляется с помощью противопоточных схем второго порядка. Связь между полями скорости и давления, обеспечивающая выполнение уравнения неразрывности, реализуется при помощи SIMPLEC-процедуры на совмещенных сетках [12]. Полученные в результате дискретизации исходной системы разностные уравнения решаются итерационным способом с применением алгебраического многосеточного решателя. В рамках этого подхода на боковых стенках канала задавались периодические граничные условия; таким образом, ширина канала считалась бесконечной. На верхней стенке задавались условия прилипания. Нижняя стенка представляла собой комбинацию участков текстурирования с граничными условиями прилипания и участков свободной поверхности, имитирующей воздушные полости между текстурами. На участках свободной 


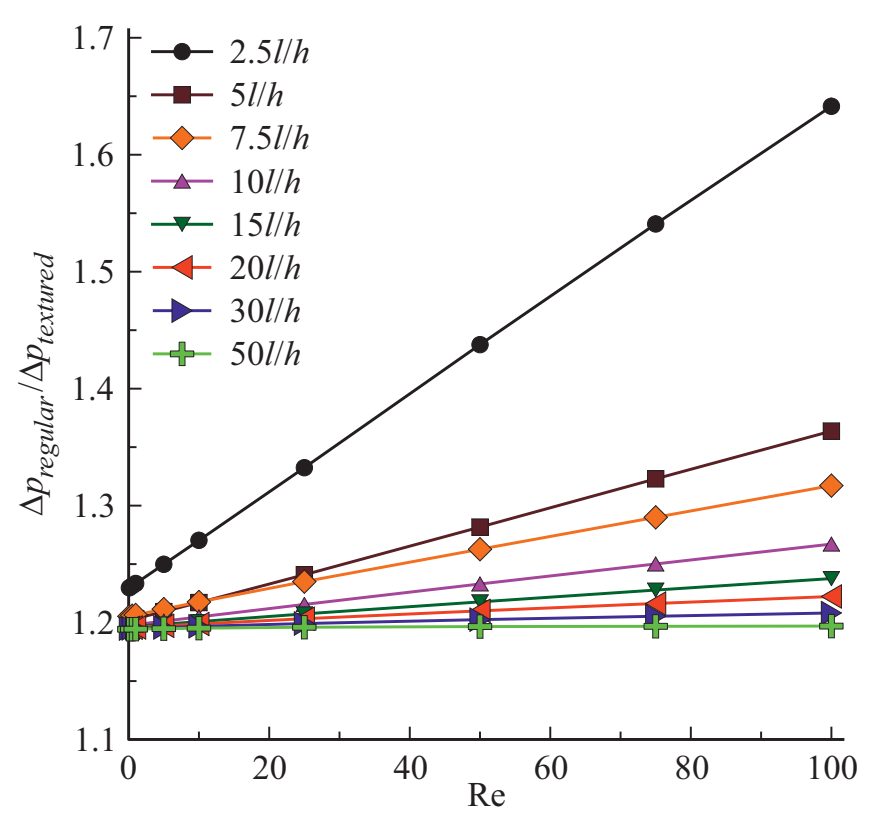

Рис. 1. Зависимость относительного перепада давлений в микроканале от числа Рейнольдса для различных относительных длин такого микроканала.

поверхности задавалось скольжение скорости потока на стенке с помощью условия равенства нулю напряжения сдвига. Участки текстурирования представляли собой квадраты со стороной $1 \mu \mathrm{m}$ и располагались в шахматном порядке с продольным и поперечным шагами, также равными $1 \mu \mathrm{m}$. На входе в микроканал задавался параболический профиль скорости, выход из канала был свободным. В качестве рабочей жидкости рассматривалась вода с вязкостью $0.001 \mathrm{~Pa} \cdot \mathrm{s}$ и плотностью $1000 \mathrm{~kg} / \mathrm{m}^{3}$. Числа Рейнольдса варьировались от 0.1 до 100 за счет изменения средней скорости потока.

В результате расчетов было установлено, что перепад давлений в текстурированном микроканале был меньше, чем в обычном канале для любой его длины и для любых чисел Рейнольдса. Вследствие этого влияние наличия текстур на стенке микроканала можно оценить с помощью безразмерного отношения перепада давлений в канале без текстурированных стенок к перепаду давлений в канале с текстурированной стенкой. Зависимость такого безразмерного критерия от числа Рейнольдса для различных безразмерных длин канала (длина канала, отнесенная к его высоте) приведена на рис. 1. Видно, что относительный перепад давлений линейно зависит от числа Рейнольдса, причем с увеличением относительной длины канала уменьшается наклон этой прямой. Видимо, это связано с тем, что верхняя стенка микроканала является нетекстурированной, и с увеличением длины канала на ней увеличиваются потери на трение. Кроме того, даже для самого длинного канала, где относительный перепад давлений не зависит от числа Рейнольдса, его значение составляет порядка 1.2 , т.е. применение текстурированной стенки позволяет снизить гидродинамические потери в таком микроканале на $20 \%$.

Другой не менее важной характеристикой является эффективная длина скольжения скорости на стенке, которая определяется как отношение средней скорости на стенке к среднему градиенту скорости на этой стенке: $l_{\text {eff }}=\left\langle u_{s}\right\rangle /\langle\delta u / \delta n\rangle$. Градиент скорости будет определяться исходя из реологического закона Ньютона: $\langle\tau\rangle=-\mu(\delta u / \delta n)$. В свою очередь напряжение сдвига $\langle\tau\rangle$ пропорционально перепаду давлений в канале. В итоге эффективная длина скольжения в щелевом микроканале будет определяться следующим образом: $l_{e f f}=\left(2\left\langle u_{s}\right\rangle \mu l\right) /(\Delta p h)$, где $\mu$-динамическая вязкость жидкости; $l, h$ - длина и высота канала соответственно; $\Delta p$ - перепад давлений в канале. Зависимость эффективной длины скольжения на стенке от числа Рейнольдса для различных безразмерных длин канала приведена на рис. 2. Видно, что эта величина нелинейно зависит как от числа Рейнольдса, так и от приведенной длины канала. Более подробные исследования влияния различных параметров на эту величину будут проведены позднее, однако исходя из этого графика можно определить, что среднее значение эффективной длины скольжения составляет порядка $630 \mathrm{~nm}$ или $6.3 \%$ от высоты микроканала.

Далее было исследовано влияние наличия текстурирования на стенке микроканала на коэффициент гидродинамического сопротивления, зависимость которого от приведенной длины канала при различных числах Рейнольдса представлена на рис. 3. Согласно теории [13], коэффициент трения для щелевого канала равен 24/Re, т.е. должен представлять собой прямую линию в координатах, используемых на рис. 3. Как видно, для

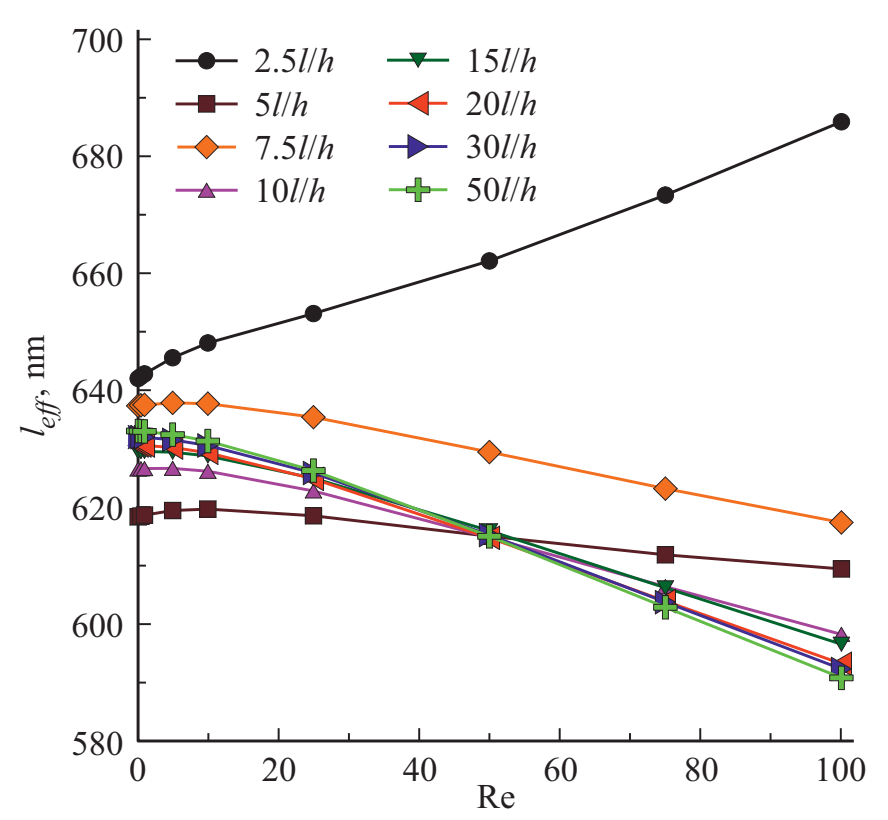

Рис. 2. Зависимость эффективной длины скольжения на стенке микроканала от числа Рейнольдса для различных относительных длин такого микроканала. 


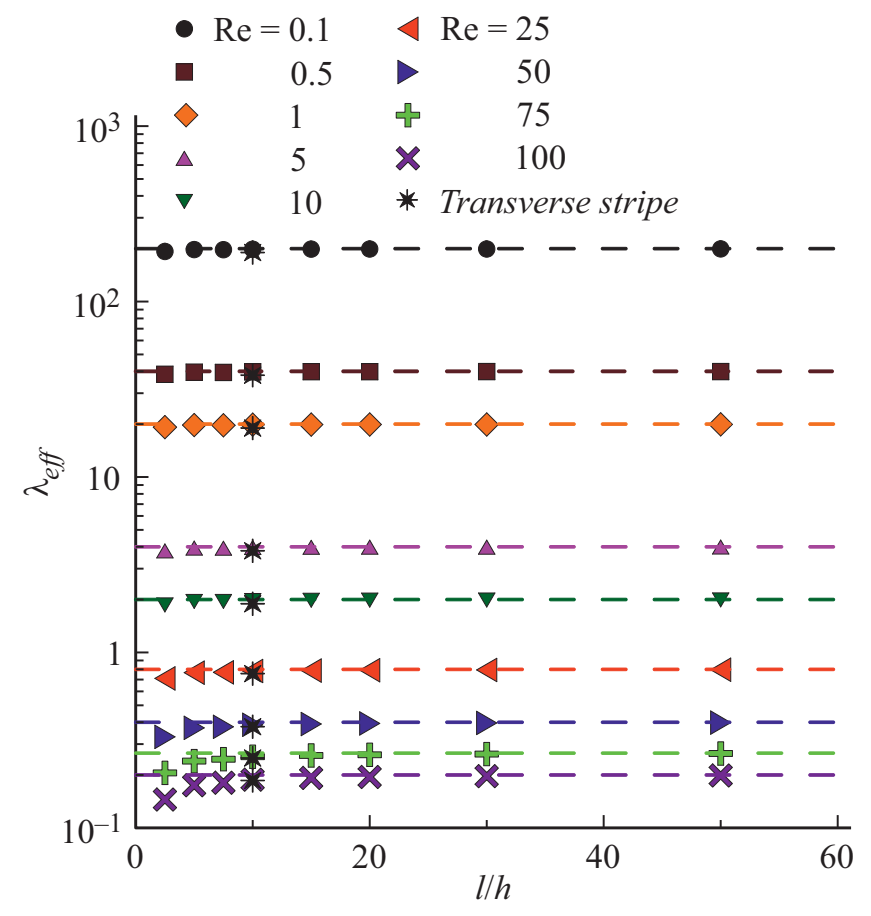

Рис. 3. Зависимость коэффициента гидродинамического сопротивления от относительной длины микроканала для различных чисел Рейнольдса.

больших значений приведенной длины микроканала эта зависимость действительно представляет собой прямую линию, однако для малых приведенных длин канала зависимость начинает отклоняться от прямолинейной, причем чем больше число Рейнольдса, тем сильнее это отклонение. В дополнение к этому даже прямые участки не соответствуют теоретическим значениям коэффициента трения: они хорошо описываются соотношением вида $\lambda_{e f f}=20 / \mathrm{Re}$, которое представлено на рис. 3 штриховыми линиями для соответствующих чисел Рейнольдса. Неудивительно, что значение коэффициента гидродинамического сопротивления для микроканала с текстурированной стенкой меньше подобной величины для микроканала с нетекстурированной стенкой на 20\%, так как это полностью согласуется с результатами исследований зависимости относительного перепада давлений, приведенными выше.

После обработки всех полученных результатов была предложена корреляция, описывающая поведение относительного перепада давлений в микроканале, следующего вида: $\Delta p_{\text {rel }}=5.69 \cdot 10^{-4}(l / h)^{-1.5} \mathrm{Re}+$ $+1.1 \cdot 10^{-2}(l / h)^{-0.8}+1.19$. При сопоставлении относительного перепада давлений, полученного с помощью этой корреляции, с результатами расчетов было обнаружено, что предложенная корреляция описывает полученные результаты с точностью более 95\%. Кроме того, для канала длиной $100 \mu \mathrm{m}$ было рассмотрено иное расположение шероховатостей: они представляли собой поперечные полосы шириной $1 \mu \mathrm{m}$ с расстоянием между ними $3 \mu \mathrm{m}$. Таким образом, площади шероховатостей и областей скольжения в такой и рассмотренной выше конфигурациях были равными. Для их количественного сравнения на рис. 3 дополнительно приведены значения коэффициента трения для шероховатости в виде поперечных полос. Видно, что с ростом числа Рейнольдса различие значений для двух рассмотренных конфигураций уменьшается, причем даже при самом маленьком числе Рейнольдса оно не превышает 5\%. Следовательно, основное влияние на снижение перепада давлений в канале оказывает относительная площадь области скольжения, а не рисунок расположения шероховатостей на поверхности.

Таким образом, в результате исследования установлено, что перепад давлений в текстурированном микроканале был меньше, чем в обычном канале для любой его длины. При этом относительный перепад давлений имеет линейную зависимость от числа Рейнольдса, причем с увеличением относительной длины канала уменьшается наклон данной прямой. Показано, что применение текстурированной стенки позволяет снизить гидродинамические потери в таком микроканале минимум на 20\%. Определен коэффициент гидродинамического сопротивления, который с достаточной степенью точности описывается корреляцией вида 20/Re. Предложена довольно простая корреляция, описывающая зависимость относительного перепада давлений от числа Рейнольдса для различных безразмерных длин канала с точностью более 95\%. Получена зависимость эффективной длины скольжения на стенке от числа Рейнольдса для различных безразмерных длин канала. Среднее значение этой величины составляет порядка $630 \mathrm{~nm}$ или 6.3\% от высоты канала. Установлено, что эффективная длина скольжения нелинейно зависит как от числа Рейнольдса, так и от приведенной длины канала. Однако чтобы ответить на вопрос о том, каковы механизмы влияния различных параметров на эту величину, необходимо провести дополнительные систематические исследования.

\section{Финансирование работы}

Исследование выполнено при финансовой поддержке Российского научного фонда в рамках проекта № 2079-00231 „Исследование возможности управления ультрагидрофобными свойствами стенок микроканалов с целью повышения энергоэффективности микрофлюидного оборудования“.

\section{Конфликт интересов}

Авторы заявляют, что у них нет конфликта интересов. 


\section{Список литературы}

[1] B. Bhushan, Y.C. Jung, K. Koch, Phil. Trans. Roy. Soc. A, 367 (1894), 1631 (2009). DOI: 10.1098/rsta.2009.0014

[2] G. Bhutani, K. Muralidhar, S. Khandekar, Interfac. Phenom. Heat Transfer, 1 (1), 29 (2013).

DOI: $10.1615 /$ InterfacPhenomHeatTransfer.2013007038

[3] H. Liu, L. Feng, J. Zhai, L. Jiang, D. Zhu, Langmuir, 20 (14), 5659 (2004). DOI: $10.1021 / 1 \mathrm{a} 036280$ o

[4] H.B. Eral, D.J.C.M. 't Mannetje, J.M. Oh, Colloid Polym. Sci., 291, 247 (2013). DOI: 10.1007/s00396-012-2796-6

[5] M.T.Z. Myint, G.L. Hornyak, J. Dutta, J. Colloid Interface Sci., 415, 32 (2014). DOI: 10.1016/j.jcis.2013.10.015

[6] A.I. Ageev, I.V. Golubkina, A.N. Osiptsov, Phys. Fluids, 30 (1), 012102 (2018). DOI: 10.1063/1.5009631

[7] A.I. Ageev, A.N. Osiptsov, J. Phys.: Conf. Ser., 1141, 012134 (2018). DOI: 10.1088/1742-6596/1141/1/012134

[8] А.Э. Муслимов, А.Ш. Асваров, Н.С. Шабанов, В.М. Каневский, Письма в ЖТФ, 46 (19), 15 (2020). DOI: 10.21883/PJTF.2020.19.50037.18371

[9] A.I. Ageev, A.N. Osiptsov, Fluid Dyn., 54 (2), 205 (2019). DOI: $10.1134 / \mathrm{S} 0015462819020010$

[10] E.S. Asmolov, T.V. Nizkaya, O.I. Vinogradova, Phys. Rev. E, 98 (3), 033103 (2018). DOI: 10.1103/PhysRevE.98.033103

[11] A.S. Lobasov, A.V. Minakov, V.V. Kuznetsov, V.Y. Rudyak, A.A. Shebeleva, Chem. Eng. Process.: Process Intensific., 134, 105 (2018). DOI: 10.1016/j.cep.2018.10.012

[12] С. Патанкар, Численные методы решения задач теплообмена и динамики жсидкости (Энергоатомиздат, М., 1984).

[13] Л.Г. Лойцянский, Механика жидкости и газа (Дрофа, M., 2003). 